Pacific Journal of Mathematics

ON PROSUPERSOLVABLE GROUPS 


\section{ON PROSUPERSOLVABLE GROUPS}

\section{B. C. Oltikar and Luis Ribes}

Let $G$ be a prosupersolvable group (projective limit of finite supersolvable groups), whose order involves only finitely many primes; then we show that $G$ is topologically finitely generated iff its Frattini subgroup is open in $G$. If a prosupersolvable group $G$ is topologically finitely generated, so is each Sylow $p$-subgroup of $G$. If $G$ is a topologically finitely generated prosupersolvable group, then every subgroup $G$ of finite index is open.

1. Introduction. A profinite group is called strongly complete (cf. [1]) if it coincides with its profinite completion, i.e., if its subgroups of finite index are open. A profinite group is said to be topologically finitely generated if it contains a dense subgroup which is finitely generated as an abstract group. It is not difficult to find examples of (nontopologically finitely generated) profinite groups which are not strongly complete (cf. e.g., [6]).

$J-P$. Serre has shown (in a letter to A. Pletch) that if $G$ is a topologically finitely generated pro- $p$ group, then $G$ is strongly complete. Since a pronilpotent group is the cartesian product of its Sylow $p$-subgroups (cf. [8]), one deduces easily the same property for pronilpotent groups whose Sylow $p$-subgroups are topologically finitely generated. M. P. Anderson (cf. [1]), has extended this result to prove that topologically finitely generated profinite groups which are extensions of a proabelian group by a pronilpotent group are also strongly complete.

In this paper we show that topologically finitely generated prosupersolvable groups are strongly complete. First we study the structure of the Frattini subgroup and the Sylow $p$-subgroups of a prosupersolvable group. One knows that if $P$ is a pro- $p$ group, then it is topologically finitely generated iff its Frattini subgroup is open. In Theorem 3.8 we prove that the same characterization is valid for prosupersolvable groups whose orders involve only finitely many primes. Let $\mathscr{C}$ be a class of finite groups closed under the operations of taking subgroups, homomorphic images and extensions, and suppose that there are at least two distinct primes $p$ and $q$ dividing the order of some group in $\mathscr{C}$. Let $X$ be a set with at least two elements; then, the Sylow $p$-subgroups of the free pro- $\mathscr{C}$ group $F(X)$ on $X$, are topologically infinitely generated (cf. [4], Prop. 1.12). In contrast we prove here (Corollary 3.9) that the Sylow $p$-subgroups of a topologically finitely generated prosupersolvable group are topologically finitely generated. 
2. The Frattini subgroup of a profinite group.

2.1. Given a profinite group $G$, its Frattini subgroup $G^{*}$ (cf. [5]) is defined to be the intersection of all the maximal closed subgroups of $G$. It is clear that a maximal closed subgroup of $G$ is in fact open. As in the discrete case, $G^{*}$ is the characteristic subgroup of $G$ consisting of the nongenerators (an element $x \in G$ is a nongenerator, if whenever $G$ is the closed subgroup generated by $x$ and a subset $Y$ of $G$, then $G$ is the closed subgroup generated by $Y$ ). The following easy property will be used often: if $\varphi: G_{1} \rightarrow G_{2}$ is an epimorphism of profinite groups, then

$$
\varphi\left(G_{1}^{*}\right) \subseteq G_{2}^{*} .
$$

Proposition 2.2. Let $\left\{G_{i} \mid i \in I\right\}$ be an inverse system of profinite groups, where $(I, \leqq)$ is a directed set, and where the cannonical maps $\varphi_{i j}: G_{i} \rightarrow G_{j}, i \geqq j$, are epimorphisms. Then

$$
\left(\lim _{\longleftarrow} G_{i}\right)^{*}=\lim _{\leftarrow} G_{i}^{*}
$$

Proof. Put $G=\lim G_{i}$, and let $\pi_{i}: G \rightarrow G_{i}$ be the canonical epimorphism (cf. [3], p. $\overleftarrow{89}$ ). As we have pointed out in 2.1, $\pi_{i}\left(G^{*}\right) \cong$ $G_{i}^{*}$; and since $G^{*}=\lim \pi_{i}\left(G^{*}\right)$, we get $G^{*} \cong \lim G_{i}^{*}$. Conversely, let $x=\left(x_{i}\right) \in \lim G_{i}^{*}$, and $\overleftarrow{\text { suppose }} x \notin G^{*}$. Then there is a maximal open subgroup $\overleftarrow{M}$ of $G$ with $x \notin M$. Hence, there is some $i \in I$ with $x_{i} \notin$ $\pi_{i}(M)$. Since $\pi_{i}(M)$ is maximal in $G_{i}$, one has $x_{i} \notin G_{i}^{*}$; a contradiction. Thus $x \in G^{*}$, i.e., $\lim G_{i}^{*} \cong G^{*}$.

The following results can now be easily deduced from the corresponding properties of finite groups.

COROLLARY 2.3. Let $G$ be a profinite group. Then

$$
G / G^{*}=\lim _{\leftarrow}(G / U) /(G / U)^{*},
$$

where $U$ runs through the open normal subgroups of $G$.

CoROLlaRY 2.4. For any profinite group $G, G^{*}$ is pronilpotent.

COROLlaRY 2.5. If $H$ is a closed normal subgroup of a profinite group $G$, then $H^{*} \cong G^{*}$.

3. Prosupersolvable groups. A projective limit of finite supersolvable groups is called prosupersolvable.

A finite group is supersolvable iff its maximal subgroups have 
prime index (cf. [10], 9.3.8). From this one deduces immediately the following

Proposition 3.2. A profinite group $G$ is prosupersolvable iff every maximal closed subgroup of $G$ has prime index.

From Corollary 3.2 and the fact that the Frattini factor of a finite supersolvable group is metabelian (cf. [10], 7.4.15 and 7.2.13), one gets the following.

Proposition 3.3. Let $G$ be a prosupersolvable group. Then $G / G^{*}$ is profinite metabelian.

3.4. Let $\pi$ denote a set of prime numbers, and $\pi^{\prime}$ the set of prime numbers not in $\pi$. A supernatural number $\delta$ (cf. [11], p. I-3) is a $\pi$-number if the prime numbers dividing $\delta$ are in $\pi$. A closed subgroup $H$ of a profinite group $G$ is a $\pi$-subgroup if the order of $H$ is a $\pi$-number. If $H$ is a maximal $\pi$-subgroup of $G$, it is called a Sylow $\pi$-subgroup of $G$, and it is called a Hall $\pi$-subgroup if it is a Sylow $\pi$-subgroup and $(G: H)$ is a $\pi^{\prime}$-number. If $G$ is prosupersolvable (and hence prosolvable), every Sylow $\pi$-subgroup is a Hall $\pi$-subgroup, and any two are conjugate (cf. [2]).

Proposition 3.5. Let $\pi$ be the set of prime numbers greater than a fixed natural number $n$. Let $G$ be a prosupersolvable group. Then

(1) there is a (unique) normal Sylow $\pi$-subgroup $K$ of $G$;

(2) there is a split exact sequence of prosupersolvable groups

$$
1 \longrightarrow K \longrightarrow G \stackrel{\varphi}{\rightleftarrows} H \longrightarrow 1
$$

with $\varphi$ an open map (i.e., $G$ is the topological semidirect product of $K$ and $H$ ), where $H$ is a Sylow $\pi^{\prime}$-subgroup of $G$.

Proof. For each open normal subgroup $U$ of $G$, let $K_{U}$ be the normal Sylow $\pi$-subgroup of $G / U$ (cf. [9], p. 232). Set $K=\lim K_{U}$. Then (cf. [2], Th. 7), $K$ is a normal Sylow $\pi$-subgroup of $G$. $\overleftarrow{\text { Now, }}$ let $H$ be a Sylow $\pi^{\prime}$-subgroup such that $G=K H$ (cf. [2], Th. 13). Then $G$ is, as an abstract group, the semidirect product of $K$ and $H$. Since all the groups involved are compact, $G$ is also the topological semidirect product of $K$ and $H$. In particular $G / K$ and $H$ are topologically isomorphic and $\varphi$ is open.

Proposition 3.6. Let $G$ be a profinite group, and $H$ a normal 
Hall $\pi$-subgroup of $G$, where $\pi$ is a set of primes. Then $H^{*}=$ $H \cap G^{*}$.

Proof. By Proposition 2.2 we may assume that $G$ is finite. Since $H^{*} \cong G^{*}$ (cf. [10], 7.3.17), we have $H^{*} \subseteq H \cap G^{*}=N \triangleleft G$. We may assume $H^{*}=1$ (otherwise we reason with $G / H^{*}$ ), and we shall show $N=1$. Since $N$ is a normal nilpotent subgroup of $H$, it is contained in the Fitting subgroup of $H$. But Fitt $(H)$ is abelian since $H^{*}=1$ (cf. [10], 7.4.15). So $N$ is abelian, and since $H^{*}=1, H$ splits over $N$ (cf. [10], 7.4.14). Thus (cf. [9], IV. 7.a) $G$ splits over $N$. Say $G=N L$ with $N \cap L=1$. If $L \neq G$, let $M \supseteqq L$ be a maximal subgroup of $G$. Then $G=N M=M$, and this is impossible. Hence $L=G$ and $N=1$.

COROLLARY 3.7. Let $G$ be a prosupersolvable group whose order is divisible only by a finite number of primes. If $p$ is the largest prime dividing the order of $G$, and $P$ is the unique Sylow p-subgroup of $G$ (Proposition 3.5), then $P^{*}=P \cap G^{*}$.

THEOREM 3.8. Let $G$ be a prosupersolvable group whose order is divisible by only finitely many primes $p_{1}, p_{2}, \cdots, p_{r}$. Then $G$ is topologically. finitely generated iff $G^{*}$ is open.

Proof. By Proposition 3.2, all maximal closed subgroups of $G$ have prime index (in particular they are open). Suppose $G$ is topologically finitely generated; then for each prime $p$ dividing the order of $G$, there are only finitely many open subgroups of index $p$. Therefore $G^{*}$ is an intersection of finitely many open subgroups, and thus it is open. The converse is obvious.

COROLLARY 3.9. Let $G$ be a topologically finitely generated prosupersolvable group. Then for each prime number $p$, every Sylow $p$-subgroup of $G$ is topologically finitely generated.

Proof. Let $p$ be a prime number. We use the notation of Proposition 3.5 with $n=p$. Then since $\pi^{\prime}$ is a finite set, by Theorem $3.8 H^{*}$ is open in $H$. Let $S_{p}$ be the unique Sylow $p$-subgroup of $H$. By Corollary 3.7, $S_{p}^{*}=S_{p} \cap H^{*}$. Then $S_{p}^{*}$ is open in $S_{p}$, and hence $S_{p}$ is topologically finitely generated. Finally, it is clear that $S_{p}$ is also a Sylow $p$-subgroup of $G$.

4. Subgroups of finite index of prosupersolvable groups.

LEMmA 4.1. Let $G$ be a profinite group and $n$ natural number 
relatively prime to the order of $G$. Then $G^{n}=G$, where $G^{n}$ is the set of $n$th powers of the elements of $G$.

Proof. The result is obviously true if $G$ is finite. Hence $(G / U)^{n}=$ $G / U$ for each open normal subgroup $U$ of $G$; i.e., the map

$$
G / U \longrightarrow G / U \text {, }
$$

given by $x \rightarrow x^{n}$ is onto. Since $G=\lim G / U$, and $\lim$ preserves ontoness (cf. [7], p. 35), we have that the map

$$
G \longrightarrow G
$$

given by $x \rightarrow x^{n}$, is also onto, i.e., $G=G^{n}$.

THEOREM 4.2. Let $G$ be a topologically finitely generated prosupersolvable group. Then every subgroup $V$ of $G$ of finite index is open, i.e., $G$ is strongly complete.

Proof. We may assume that $V$ is normal in $G$ (otherwise we work with the intersection of the conjugates $V$ in $G)$. Say $(G: V)=$ $n$. Let $\pi$ consist of the prime numbers greater than $n$. Let $K$ be the Sylow $\pi$-subgroup of $G$; then we have a split exact sequence of profinite groups

$$
1 \longrightarrow K \longrightarrow G \stackrel{\varphi}{\rightleftarrows} H \longrightarrow 1
$$

where $H$ is a Sylow $\pi^{\prime}$-subgroup of $G$ (Proposition 3.5). Then $n$ and the order of $K$ are relatively prime. Hence (Lemma 4.1) $K=K^{n}$, and so $K \subseteq V$. Therefore $V=\varphi^{-1}(\varphi V)$. Thus it suffices to show that $\varphi V$ is open in $H$. Now, $H$ is topologically finitely generated prosupersolvable and its order involves finitely many primes only. Hence, by Theorem 3.8 its Frattini subgroup $H^{*}$ is open in $H$. Therefore $H^{*}$ is topologically finitely generated. On the other hand $H^{*}$ is pronilpotent (Corollary 2.4). As we have pointed out in the Introduction (also, cf. [1], p. 26), it follows then from Serre's result that every subgroup of $H^{*}$ of finite index is open. Therefore, $(\varphi V) \cap H^{*}$ is open in $H^{*}$, which in turn is open in $H$; hence $(\varphi V) \cap H^{*}$ is open in $H$. Since $\varphi V$ contains an open subgroup of $H$, it is also open in $H$.

\section{REFERENCES}

1. M. P. Anderson, Subgroups of finite index in profinite groups, Pacific J. Math., 62 (1976), 19-28.

2. E. D. Bolker, Inverse limits of solvable groups, Proc. Amer. Math. Soc., 14 (1963), 147-152.

3. N. Bourbaki, General Topology, Addison-Wesley, Reading, Mass., 1966. 
4. D. Gildenhuys and C.-K. Lim, Free pro-CE-groups, Math. Z., 125 (1972), 233-254.

5. K. W. Gruenberg, Projective profinite groups, J. London Math. Soc., (3) 42 (1967), $155-165$.

6. H. L. Peterson, Discontinuous characters and subgroups of finite index, Pacific J. Math., 44 (1973), 683-691.

7. L. Ribes, Introduction to profinite groups and Galois cohomology, Queen's Papers in Pure and Applied Math., Kingston, Canada, 1970.

8. - Productos amalgamados de grupos pronilpotentes, Rev. Mat. Hisp.-Amer., 33 (1973), 49-53 and 133-138.

9. E. Schenkman, Group Theory, Van Nostrand, Princeton, N. J., 1965.

10. W. R. Scott, Group Theory, Prentice Hall, Englewood Cliffs, N. J., 1964.

11. J-P. Serre, Cohomologie Galoisienne, Lect. Notes Math., no. 5, Springer, Berlin, 1965.

Received May 6, 1977 and in revised form December 8, 1977.

Carleton University

Ottawa, Ont., Canada 


\section{PACIFIC JOURNAL OF MATHEMATICS}

\section{EDITORS}

RICHARD ARENS (Managing Editor)

University of California

Los Angeles, California 90024

C. W. Curtis

University of Oregon

Eugene, OR 97403

C. C. MOORE

University of California

Berkeley, CA 94720

\section{J. DUGUNDJI}

Department of Mathematics University of Southern California Los Angeles, California 90007

R. Finn AND J. Milgram Stanford University Stanford, California 94305

\section{ASSOCIATE EDITORS}

E. F. BeCK ENBACH

B. H. NeUMaNN

F. WOLF

K. Yoshida

\section{SUPPORTING INSTITUTIONS}

UNIVERSITY OF BRITISH COLUMBIA CALIFORNIA INSTITUTE OF TECHNOLOGY UNIVERSITY OF CALIFORNIA MONTANA STATE UNIVERSITY UNIVERSITY OF NEVADA, RENO NEW MEXICO STATE UNIVERSITY OREGON STATE UNIVERSITY UNIVERSITY OF OREGON
UNIVERSITY OF SOUTHERN CALIFORNIA STANFORD UNIVERSITY UNIVERSITY OF HAWAII UNIVERSITY OF TOKYO UNIVERSITY OF UTAH WASHINGTON STATE UNIVERSITY UNIVERSITY OF WASHINGTON 


\section{Pacific Journal of Mathematics \\ Vol. 77, No. $1 \quad$ January, 1978}

Dan Amir, Chebyshev centers and uniform convexity ............... 1

Lawrence Wasson Baggett, Representations of the Mautner group. I ..... 7

George Benke, Trigonometric approximation theory in compact totally

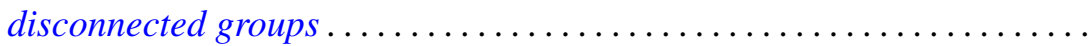

M. Bianchini, O. W. Paques and M. C. Zaine, On the strong compact-ported topology for spaces of holomorphic mappings ..................

Marilyn Breen, Sets with $(d-2)$-dimensional kernels

J. L. Brenner and Allen Kenneth Charnow, Free semigroups of $2 \times 2$

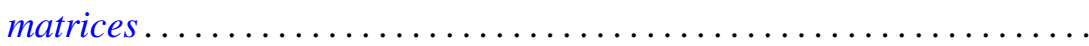

David Bressoud, A new family of partition identities .................

David Fleming Dawson, Summability of matrix transforms of stretchings

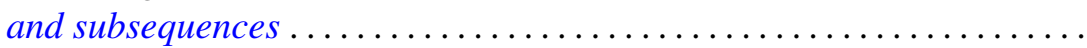

Harold George Diamond and Paul Erdôs, A measure of the nonmonotonicity

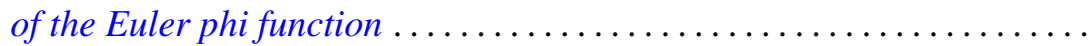

Gary Doyle Faulkner and Ronald Wesley Shonkwiler, Kernel dilation in reproducing kernel Hilbert space and its application to moment

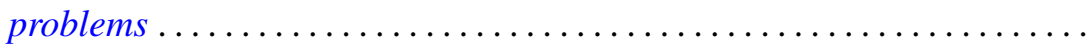

Jan Maksymilian Gronski, Classification of closed sets of attainability in the

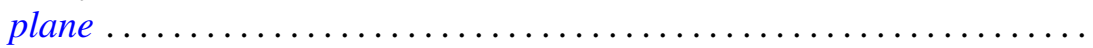

H. B. Hamilton and T. E. Nordahl, Semigroups whose lattice of congruences

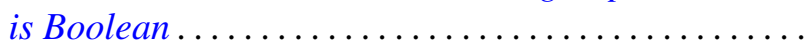

Harvey Bayard Keynes and D. Newton, Minimal $(G, \tau)$-extensions ...

Anthony To-Ming Lau, The Fourier-Stieltjes algebra of a topological

semigroup with involution.

B. C. Oltikar and Luis Ribes, On prosupersolvable groups ...

Brian Lee Peterson, Extensions of pro-affine algebraic groups ...

Thomas M. Phillips, Primitive extensions of Aronszajn spaces ...

Mehdi Radjabalipour, Equivalence of decomposable and 2-decomposable operators. .

M. Satyanarayana, Naturally totally ordered semigroups .

Fred Rex Sinal, A homeomorphism classification of wildly imbedded two-spheres in $S^{3}$

Hugh C. Williams, Some properties of a special set of recurring

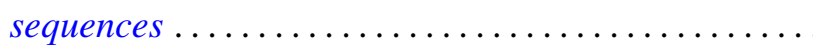

